S.I. GOLDBERG

KŌDAI MATH. SEM. REP

28 (1977) $211-213$

\title{
CURVATURE AND REAL ANALYSIS
}

\author{
By SAMuel I. GoldBerg ${ }^{1}$
}

1. Introduction. In a recent paper with S.-S. Chern [3], the author studie the volume decreasing property of a class of harmonic mappings thereby obtain ing a real analogue of the classical Schwarz-Ahlfors lemma. The domain $\Lambda$ was taken to be the unit open ball with the hyperbolic metric of constan negative curvature, and the image space was a negatively curved Riemannia: manifold with sectional curvature bounded away from zero. In this paper, i is shown that $M$ may by taken to be any complete Riemannian manifold o nonpositive curvature provided its sectional curvatures are bounded below $b$ : a negative constant (see [5]). The technique employed also yields a distanc decreasing theorem when the map is volume preserving.

2. Harmonic mappings. Let $M$ and $N$ be $C^{\infty}$ oriented Riemannian manifold: of the same dimension $n$ with metrics $d s_{M}{ }^{2}$ and $d s_{N}{ }^{2}$, respectively, and volum elements $d v_{M}$ and $d v_{N}$. Let $f: M \rightarrow N$ be a $C^{\infty}$ mapping and $A=f^{*} d v_{N} / d v_{M}$ be th ratio of volume elements. We calculate the Laplacian $\Delta$ of $u=A^{2}$ as in [3] and so recall the necessary Riemannian geometry. Locally, then, $d s_{M}{ }^{2}=\sum \omega_{i}$ and $d s_{N}{ }^{2}=\Sigma \omega_{a}{ }^{* 2}$, where the $\omega_{i}$ and $\omega_{a}{ }^{*}$ are linear differential forms in $M$ anc $N$, respectively. The structure equations in $M$ are

$$
d \omega_{i}=\sum_{\jmath} \omega_{\jmath} \wedge \omega_{j i}, d \omega_{i j}=\sum_{k} \omega_{i k} \wedge \omega_{k \jmath}-\frac{1}{2} \sum_{k, l} R_{\imath j k l} \omega_{k} \wedge \omega_{l} .
$$

The Ricci tensor is defined by $R_{\imath j}=\sum_{k} R_{i k j k}$, and the scalar curvature by $R=$ $\sum_{i} R_{i i}$. (The corresponding quantities in $N$ will be denoted with an asterisk.)

Let $f^{*}: \Lambda(N) \rightarrow \Lambda(M)$ be the pull-back map, and set $f^{*} \omega_{a}{ }^{*}=\sum_{i} A_{\imath}{ }^{a} \omega_{i}$. (Ir the sequel, we will drop $f^{*}$ from such formulas when its presence is clear from the context.) The covariant differential of the tangent mapping $f_{*}$ is definec by

Received Oct. 23, 1975.

This is a summary of a portion of a lecture given at the Tokyo Institute of Technology on August 15, 1975.

1 This research was supported in part by the National Science Foundation. 


$$
d A_{\imath}{ }^{a}+\sum_{\jmath} A_{\jmath}{ }^{a} \omega_{j i}+\sum_{b} A_{\imath}{ }^{b} \omega_{b a}{ }^{*} \equiv \sum_{\jmath} A_{\imath \jmath}{ }^{a} \omega_{\jmath} \quad \text { (say) }
$$

where $A_{\imath \jmath}{ }^{a}=A_{j i}{ }^{a}$. The mapping $f$ is called harmonic if $\sum_{i} A_{i i}{ }^{a}=0$. The following formula for the Laplacian $\Delta$ of $u$ was obtained in [3]:

$$
\frac{1}{2} \Delta u=2 \sum_{\jmath}\left(A_{\jmath}\right)^{2}-C+A \sum_{a, \imath, j} B_{a}{ }^{\imath} A^{a}{ }_{j j i}+u\left(R-\sum_{b, c, j} R_{b c} * A_{\jmath}{ }^{b} A_{\jmath}{ }^{c}\right),
$$

where $\left(B_{a}^{i}\right)$ is the adjoint matrix of $\left(A_{\jmath}{ }^{a}\right), C=\Sigma B_{a}{ }^{2} B_{b}{ }^{k} A_{k \jmath}{ }^{a} A_{\imath \jmath}{ }^{b}$ is a scalar invariant of the mapping, $d A=\sum A_{j} \omega_{\jmath}$, and the $A^{a}{ }^{a}{ }{ }{ }$ are defined by

$$
d A_{\imath \jmath}{ }^{a}+\sum_{b} A_{\imath \jmath}{ }^{b} \omega_{b a}{ }^{*}+\sum_{k} A_{k \jmath}{ }^{a} \omega_{k \imath}+\sum_{k} A_{i k}{ }^{a} \omega_{k \jmath} \equiv \sum_{k} A^{a}{ }_{\imath j k} \omega_{k} .
$$

The mapping $f$ is said to be totally degenerate if $u$ vanishes everywhere.

3. Distortion theorem. We sketch the proof of the following.

THEOREM. Let $M$ be a complete Riemannian manifold whose sectional curv. atures are nonpositive and bounded below by a negative constant $-A$. Let $f: M \rightarrow N$ be a harmonic mapping of equidimensional spaces of dimension $n$ satisfying the condition $C \leqq 0$. If $N$ is an Einstern space with scalar curvature $R^{*} \leqq$ $-n(n-1) A$, or if its sectional curvatures are $\leqq-A$, then $f$ is volume decreasing.

If $f$ is volume preserving and either $N$ is Einsteinian with $R^{*} \leqq-n^{2}(n-1) A$, or if its sectional curvatures are $\leqq-n A$, then it is distance decreasing.

The technique employed is to distort the metric of the domain $M$ conformally in such a way that the ratio of volume elements attains its maximum on $M$. Let $d \tilde{s}^{2}$ be a Riemannian metric of $M$ conformally related to $d s^{2}$. Then, there is a function $p>0$ on $M$ such that $d \tilde{s}^{2}=p^{2} d s^{2}$. In the sequel, we distinguish the elements of $M$ referred to $d \tilde{s}^{2}$ with a tilda. Put $d \log p=\sum p_{i} \omega_{i}$. Then, if $f$ is harmonic

$$
\begin{aligned}
\frac{1}{2} \tilde{\Delta} \tilde{u}=2 \sum_{\jmath}\left(\tilde{A}_{\jmath}\right)^{2}-\tilde{C}+(n-2) q^{2 n+2}\left[A \sum_{a, \imath, \jmath} B_{a}{ }^{2} A_{\imath \jmath}{ }^{a} p_{\jmath}+u \Delta \log p\right. \\
\left.-2 u \sum_{j}\left(p_{\jmath}\right)^{2}\right]+\tilde{u}\left(\tilde{R}-\sum_{b, c, \jmath} \tilde{A}_{\jmath}{ }^{b} A_{\jmath}{ }^{c} R_{b c} *\right), \quad q=1 / p .
\end{aligned}
$$

LEMMA 1. If $f$ is a harmonic mapping, then

$$
\widetilde{C}=q^{2 n+2}\left[C-(n-2) u \sum_{j}\left(p_{\jmath}\right)^{2}\right] .
$$

Thus, if $C$ is nonpossitive, so is $\tilde{C}$.

If $\tilde{u}$ attains its maximum at $x \in M$, then at $x$,

$$
A \sum_{a, \imath, j} B_{a}{ }^{2} A_{j i}{ }^{a} p_{\jmath}+u \Delta \log p-2 u \sum_{\jmath}\left(p_{\jmath}\right)^{2}=u\left[(n-2) \sum_{j}\left(p_{\jmath}\right)^{2}+\Delta \log p\right] .
$$

LEMMA 2. Let $f$ be harmonic with respect to $\left(d s_{M}{ }^{2}, d s_{N}{ }^{2}\right)$ with the property $C \leqq 0$, and let $\tilde{u}$ attain its maxımum at $x \in M$. If $n=2$, or if the function 
$P=(n-2) \sum_{j}\left(p_{\jmath}\right)^{2}+\Delta \log p$ is nonnegative everywhere on $M$, then either $f$ is totally degenerate, or else $-\sum_{b, c, j} R_{b c} * \tilde{A}_{\jmath}^{b} \tilde{A}_{\jmath}^{c} \leqq-\tilde{R}$ at $x$.

The remainder of the proof is due to Har'El [5] except for the method used to establish the boundedness of $\Delta \tau$. Let $y$ be a point of $M$ and denote by $d(x, y)$ the distance-from-y function. Then, $t(x)=(d(x, y))^{2}, x \in M$, is $C^{\infty}$ and convex on $M$ (see [2]). (If $M$ is not simply connected, consider its simply connected covering.) The function $\tau(x)=d(x, y)$ is also convex, but it is only continuous on $M$. The convex open submanifolds $M_{\rho}=\{x \in M \mid t(x)<\rho\}$ of $M$ exhaust $M$, that is, $M=\bigcup_{\rho<\infty} M_{\rho}$.

Consider the metric $d \tilde{s}^{2}=(\rho / \rho-t)^{2} d s^{2}$ on $M_{\rho}$. Then $\tilde{u}=(\rho-t / \rho)^{2 n} u$ is nonnegative and continuous on the closure $\bar{M}_{\rho}$ of $M_{\rho}$ and vanishes on $\partial M_{\rho}$. Since $\bar{M}_{\rho}$ is compact, $\tilde{u}$ has a maximum in $M_{\rho}$. Since $t(x)$ is convex, the function $P$ is positive, so we obtain the conclusion of Lemma 2 .

Relating the scalar curvatures $\widetilde{R}$ of $M_{\rho}$ and $R$ of $M$, we obtain

$$
\widetilde{R}=\frac{(\rho-t)^{2}}{\rho^{2}} R-2(n-1) \frac{\rho-t}{\rho} \frac{\Delta t}{\rho}-4 n(n-1) \frac{t}{\rho^{2}}, \quad t<\rho .
$$

LEMMA 3. For each $\rho$, there exists a positive constant $\varepsilon(\rho)$ such that the inequality

$$
\widetilde{R} \geqq-n(n-1) A-\varepsilon(\rho)
$$

holds om $M(\rho)$. Moreover $\varepsilon(\rho) \rightarrow 0$ as $\rho \rightarrow \infty$.

To see that $\Delta \tau$ is bounded as $\tau \rightarrow \infty$, observe that the level hypersurfaces of $\tau$ are spheres $S$ with $y$ as center. The hessian of $\tau$ can be identified with the second fundamental form $h$ of those spheres, extended to be 0 in the normal direction. It follows that $\Delta \tau=$ trace $h=(n-1) \cdot$ mean relative curvature of $S$. If the curvature $K \geqq a^{2}$, then from [1; pp. 247-255], $\Delta \tau \leqq(n-1) a \cot a \tau$. If we put $a^{2}=-\alpha^{2}$, then $\Delta \tau \leqq(n-1) \alpha \operatorname{coth} \alpha \tau$.

The theorem is now a consequence of Lemmas $1-3$.

\section{BIBLIOGRAPHY}

[1] R. L. Bishop And R. J. CRittenden, “Geometry of Manifolds", Academic Press Inc., New York, 1964.

[2] R. L. Bishop AND B. O’Neill, Manifolds of negative curvature, Trans. Amer. Math. Soc. 145 (1969), 1-49.

[3] S.-S. Chern And S. I. GoldberG, On the volume-decreasing property of a class of real harmonic mappings, Amer. J. Math., 97 (1975), 133-147.

[4] S. I. GoldBerg, On the distance-decreasing property of a class of real harmonic mappirgs, Geometriae Dedicata, 4 (1975), 61-69.

[5] Z. HAR'EL, Harmonic mappings and distortion theorems, thesis, Technion, Israel Inst. of Tech., Haifa, 1975.

UNIVERSITY OF ILLINOIS

URBANA, IllinoIS 61801 